\title{
THE CIRCULAR RFQ STORAGE RING *
}

\section{A. G. Ruggiero", Brookhaven National Laboratory, PO BOX 5000, Upton, NY 11973}

\begin{abstract}
This paper presents a novel idea of storage ring for the accumulation of intense beams of light and heavy ions at low energy. The new concept is a natural development of the combined features of conventional storage rings and ion traps, and is basically a linear RFQ bent on itself. The advantages are: smaller beam dimensions, higher beam intensity, and a more compact storage device.
\end{abstract}

\section{INTRODUCTION}

There is need to develop compact storage rings for the accumulation of low-energy beams of ions for a variety of applications: molecular and atomic physics, solid state, chemical-physics, astrophysics, and other more exotic applications like crystalline beams and ion fusion for energy production. Several experiments can be conceived which make use of these compact storage rings: colliding beams circulating in two intersecting storage rings, collision of a stored beam of ions with an internal target, head-on collision with an electron beam or a X-ray beam from a synchrotron radiation source. Relatively high beam intensity is required. Small beam dimensions can be achieved with techniques like electron and laser cooling. In this paper we describe a novel idea of a compact storage ring for the accumulation of intense beams of light and heavy ions. The concept takes advantages of established principles of conventional low-energy storage rings, ion traps, and RFQ's. The proposed new storage ring is basically a circular RFQ bent on itself and closed mechanically. Instead of quadrupole magnets, focusing of the particles is provided by the if field of the structure. Since electrically the structure is not closed on itself, it is expected that ion beams can be stored at intensities higher than those achieved in conventional storage rings.

\section{A CONVENTIONAL STORAGE RING}

An example of a low-energy and small storage ring is ASTRID [1], used for the accumulation of light and heavy ions and for the demonstration of Laser Cooling. The ring magnetic rigidity is $1.87 \mathrm{~T}-\mathrm{m}$. The circumference is about $40 \mathrm{~m}$. It is made of eight dipole magnets which bend the beam trajectory on a circular and closed orbit and sixteen quadrupole magnets for transverse focusing. There are four periods, each with a straight section about 4 meter

\footnotetext{
- Work performed under the auspices of the US DoE

'Email:agr@bnl.gov
}

long. There is a limit on the intensity that can be stored in a conventional storage ring given by the space-charge tune-depression

$$
\Delta \nu=N Q^{2} r_{0} / 2 A \beta^{2} \gamma^{3} \varepsilon
$$

where $r_{0}=1.535 \times 10^{-18} \mathrm{~m}$ is the classical proton radius, $\mathrm{Q}$ the ion charge state, $A$ its mass number, $\beta$ and $\gamma$ respectively the velocity and energy relativistic factors, $N$ the number of ions stored, and, finally, $\varepsilon$ is the beam emittance. The tune-depression $\Delta v$ cannot exceed a value of at most 0.5 . This limit is understood to be set by the presence of unavoidable random magnet imperfections which cause the creation of stopbands around the halfintegral values of the betatron tune. A more relevant parameter is the beam transverse density in the physical space $D=N / S$, where $S=\pi a^{2}$ is the beam cross-section area. There is a relation between the beam radius $a$ and the emittance $\varepsilon$, so that

$$
S=\beta_{L} \varepsilon
$$

The average value of the amplitude lattice function $\beta_{\mathrm{L}}$ for ASTRID is about 5 meter. In a conventional storage ring, the amplitude lattice function $\beta_{\mathrm{L}}$ is a measure of the strength of focusing. The average value of $\beta_{\mathrm{L}}$ is given roughly by the length of the focusing period. Unfortunately, in a conventional storage ring, the average value of $\beta_{\mathrm{L}}$ can hardly be less than few meters; in fact, quadrupoles have a significant length, and space between quadrupoles is required to accommodate several devices. In conclusion, the physical density that can be achieved in a conventional low-energy storage ring is limited, first, by the largest amount of space-charge tune-depression according to Eq. 1, and, second, by the strength of focusing according to Eq. 2.

\section{THE LINEAR RFQ}

Another accelerator device [2] is the Radio-Frequency Quadrupole (RFQ). The device accelerates quickly the beam from an ion source to energies large enough when space-charge effects are considerably reduced, and provides simultaneously focusing of the transverse motion of the particles. The RFQ is not a magnetic device, but employs an alternating of electric field for both the acceleration and the focusing of the particles. It is a straight waveguide with four internal metallic rods. A if 
voltage at the frequency $f=\omega / 2 \pi$ is applied between diagonally opposite electrodes to generate in the opening a quadrupolar oscillating field of the same frequency. The motion of the particle will modulate the actual field in time, creating the equivalent of an alternating focusing transverse field with periodicity $L=\beta \lambda$, where $\lambda=2 \pi \mathrm{c} / \omega$ is the if wavelength. If acceleration is not required, but only transverse focusing, which is the case of interest here, the electrodes will appear just straight. In this case the excitation is a of wave oscillating stationary between the pairs of electrodes.

\section{THE ION TRAP}

Recently, a quadrupole storage ring [3] has been used for the study of ion crystalline structures. The diameter of the device is of only $12 \mathrm{~cm}$. It is made of four annular electrodes with an internal diameter of only $5 \mathrm{~mm}$. An electrostatic voltage is applied between each pair of diagonally opposing electrodes. This generates a constant radial electric field, which vanishes at the center, and increases about linearly with the distance from the main axis. An atomic gas of the desired ion species is diffused in the region between the electrodes. An electron gun ionizes the atoms, and the resulting ions are trapped transversely in the small storage ring, oscillating around the circular main axis under the effect of the restoring forces of the electrostatic quadrupole. There is no beam in this configuration since the ions do not drift azimuthally along the main axis. The particles adjust their mutual longitudinal distance by Coulomb interaction, whereas the external quadrupolar forces compensate the transverse interaction.

\section{THE CRFQ CONCEPT}

The Circular Radio-Frequency Quadrupole (CRFQ) is a compact storage ring, which includes features of all the devices described above. It is a storage ring where beams of light and heavy ions (including protons and negative ions) can circulate at constant speed corresponding to energies comparable to those used for ASTRID. Like in the Ion Trap, four annular electrodes provide focussing. Like in the linear RFQ, if oscillating voltages are applied between the electrodes to provide transverse altemating focusing of motion over a short period of about few centimeters or less. From this point of view, the CRFQ resembles the linear RFQ without acceleration, and without, therefore, corrugated vanes. The CRFQ is actually a linear $R F Q$ curved and closed mechanically on it as shown in Figs. 1 and 2.

\section{EQUATIONS OF MOTION}

It is required to keep the motion of the ions focused and on a circular orbit, in proximity of the azimuthal axis of the CRFQ. We shall assume that the major radius $\mathrm{R}$ of the $\mathrm{CRFQ}$ is much larger than the focusing period $\mathrm{L}$, and that this is in turn larger than the internal diameter $2 b$. The equations of motion are (see Fig. 1 for the orientation of $x, y)$

$$
\begin{gathered}
\mathrm{d}^{2} \mathrm{y} / \mathrm{ds^{2 }}+\left(\mathrm{B} / \mathrm{L}^{2}\right) \mathrm{x} \cos (\mathrm{ks})=0 \\
\mathrm{~d}^{2} \mathrm{x} / \mathrm{ds^{2 }}+\left(\mathrm{B} / \mathrm{L}^{2}\right) \mathrm{y} \cos (\mathrm{ks})= \\
{\left[1-\mathrm{B}(\mathrm{b} / \mathrm{L})^{2} \cos (\mathrm{ks})\right] / \mathrm{R}}
\end{gathered}
$$

where $s$ is the longitudinal path length, and

$$
B=Q e V_{0} \lambda^{2} / A m c^{2} b^{2}
$$

It can be seen that the motion is kept on a circular trajectory by the extra term due to the curvature of the restoring forces. All the considerations made for the linear RFQ apply also to the CRFQ. For instance, the $B$ parameter determines the phase advance per period and the amplitude lattice function $\beta_{\mathrm{L}} \sim \mathrm{L}$. As an example, the phase advance of $90^{\circ}$ requires $B=6.812$. Thus, the Circular Radio-Frequency Quadrupole Storage Ring has the advantage to provide a very short focusing alternating period considerably smaller than that can be obtained in a conventional storage ring.

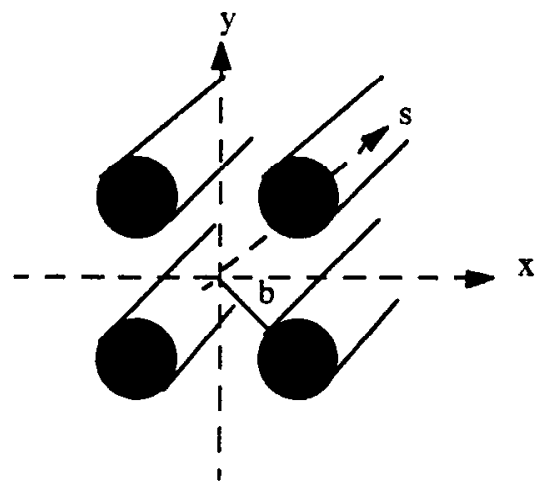

Figure 1. Cross-Section of a CRFQ

\section{SPACE-CHARGE LIMIT}

The other major advantage for the use of the CRFQ storage ring is the larger beam intensity that can be stored compared to that of a conventional storage ring of the same energy. In the latter there is the limit caused by the Space-Charge Tune-Depression $\Delta v$ that cannot exceed a value of at most 0.5 . In the CRFQ the situation is different and identical to that encountered in the linear RFQ. First, the focusing periodicity is very high and cannot be broken down by magnetic imperfections that do not exist. Secondly, the structure, though mechanically closed on it, is electrically open. Turn after turn, the CRFQ is just like a long transport. One can then make the analogy with the linear RFQ where the Space-Charge limit is caused by lowering of the phase advance per period from $90^{\circ}$ down to $45^{\circ}$ (or less), below which the particle motion may become unstable. The equations of motion are then to be 
modified by adding a space-charge term with the parameter

$$
\Delta=Q^{2} N r_{0} \lambda^{2} / A \pi^{2} R
$$

The depression of the phase advance of a single period due to space charge forces from $90^{\circ}$ down to $45^{\circ}$ corresponds to $\Delta \sim 0.044$.

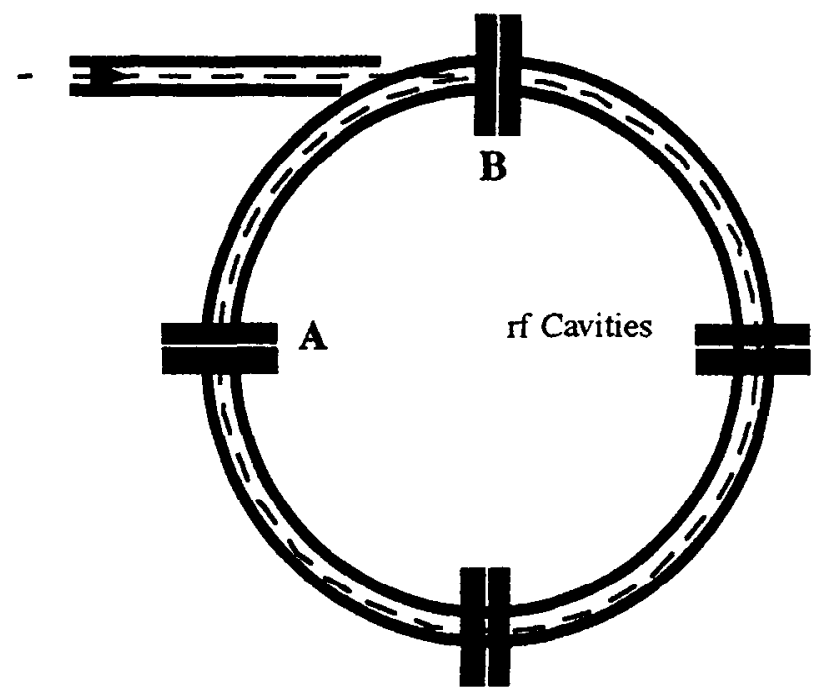

Figure 2. Plan View of a CRFQ

\section{RF POWER SOURCE}

Powering of a linear four-rod RFQ structure has been discussed by Hutcheon [4]. We follow essentially the same approach, which requires placing a number of cavities around the ring as shown in Fig. 2. These cavities function as if couplers by providing the needed if voltage difference between the two pairs of opposite rods. The analysis of Hutcheon had to be extended to include the geometric closure of the CRFQ.

\section{INJECTION}

Injection of a single beam pulse is possible if the duration is sufficiently shorter than the revolution period in the ring. During injection, the $f f$ is turned off in a section of the CRFQ as shown in Fig. 2, between A and B. This section is mechanically cut out and electrically isolated from the rest of the ring. In the remaining of the ring the if is kept on. Once the tail of the beam has entered the energized section, then also the isolated section is turned on in a period of time about the duration of the beam gap.

\section{AN EXAMPLE}

The main purpose of this example is the construction of a prototype to demonstrate the two basic principles: (i) that it is possible to achieve very short alternating focusing periods of few centimeters, and (ii) that it is possible to store ion beams at intensities higher than those that can be achieved in conventional storage rings. We take a proton beam at $100 \mathrm{keV}$ and a if of $200 \mathrm{MHz}$. The ring radius is $\mathrm{R}=50 \mathrm{~cm}$, the peak of voltage $\mathrm{V}_{0}=160 \mathrm{kV}$, the internal diameter $2 b=15 \mathrm{~mm}$. The density which corresponds to the RFQ space-charge limit is $D=2 \times 10^{12} \mathrm{~cm}^{-2}$. With a beam normalized emittance of $1 \pi \mathrm{cm} \mathrm{mrad,} \mathrm{the} \mathrm{average}$ beam radius at the space-charge limit is a $=5 \mathrm{~mm}$, so that about $2 \times 10^{12}$ protons can be stored in the ring. This is about 10 times larger than the conventional limit given by Eq 1. Thus an experiment can be done to verify that indeed it is possible to store considerable more current in the CRFQ storage ring. The circulating current at the RFQ space-charge limit is $460 \mathrm{~mA}$, and only $50 \mathrm{~mA}$ with the conventional limit of Eq 1. An ion source, operating at a very low duty cycle, is certainly capable to produce a beam pulse of about $0.5 \mu$ s duration in excess of $100 \mathrm{~mA}$. The pulse duration is shorter than the revolution period so that only one turn need to be injected in the CRFQ. The ion source can be placed on a platform at $35 \mathrm{kV}$ olt. The energy difference to $100 \mathrm{keV}$ can be obtained by accelerating the beam in a short linear RFQ operating also at $200 \mathrm{MHz}$. At the end of the acceleration, the vanes of the linear RFQ are no longer corrugated, and only focusing is then provided. This linear section would then merge the CRFQ. The experiment can be performed at a very low duty cycle, for example, with a beam pulse injected and stored in the CRFQ every few minutes. There is thus no much beam power involved and the beam itself can directly be disposed by turning off the if field.

\section{CONCLUSIONS}

We have described a new concept of Storage Ring for low-energy ion beams. The principle of operation of the new device is similar to that of an ordinary RFQ, except that it is mechanically bent on itself. It is then possible to achieve very short alternating focusing periods, and to store considerable higher beam intensity well beyond the ordinary space-charge limit of conventional storage rings.

\section{ACKNOWLEGEMENTS}

The author wishes to thank J. Alessi, M. Blaskiewicz and $D$. Raparia for very useful discussions, and $T$. Roser for continuous support.

\section{REFERENCES}

[1] S. P. Møller, IEEE 91CH3038-7, page 2811.

[2] J. W. Staples, AIP Conference Proceedings 249 (1992). Vol. II, page 1483.

[3] H. Walther, Crystalline Beams and Related Issues. Page 149. The Science and Culture Series - Physics 11 (1996). Editors D. M. Maletic and A.G. Ruggiero. World Scientific.

[4] R.M. Hutcheon, Proc. Linac Confer. 1994, page 94. 